\title{
APPLICATION OF LINEAR SIX-PARAMETER SHELL THEORY TO THE ANALYSIS OF ORTHOTROPIC TENSEGRITY PLATE-LIKE STRUCTURES
}

\author{
Paulina Obara \\ Kielce University of Technology, Faculty of Civil Engineering and Architecture, Kielce, Poland \\ e-mail: paula@tu.kielce.pl
}

Application of the linear six-parameter shell theory to the analysis of orthotropic tensegrity plate-like structures is proposed in the paper. A continuum model of a tensegrity plate with the self-stress state included is used. The tensegrity module, which is based on 4-strut expanded octahedron modules with additional connecting cables is proposed as an example. Different planes of support of the structures are taken into account and thus different reference surfaces of the plate model are considered. The self-stress state and some geometrical parameters are introduced for parametric analysis.

Keywords: linear six-parameter shell theory, tensegrity plate-like structures, self-stress state

\section{Introduction}

The concept of tensegrity structures covers trusses consisting of isolated compressed elements (struts) inside a continuous net of tensioned members (cables) (Motro, 2003; Skelton and Oliveira, 2009). The specificity of these structures lies in infinitesimal mechanisms balanced with self-stress states. Tensegrity as a structural system offers many advantages over conventional structural systems. Proper actuation can keep it stiff during deployment without requiring external members, which is the main benefit of the system. The tensegrity concept has found applications in civil engineering structures such as towers (Schlaich, 2004; Gilewski et al., 2015), bridges (Gilewski and Kasprzak, 2011) and domes (Gómez-Jáuregui, 2010). Tensegrity based on spatial reticulated systems are double-layer tensegrity grids (Gómez-Jáuregui et al., 2012) with two parallel horizontal networks of members in tension forming the top and bottom layers. The grid nodes are linked by vertical and inclined bracing members in compression and tension. These systems can be treated as tensegrity plate-like structures. Examples are Kono's structure (Kono et al., 1999) and Blur building (Crawfordt, 2016; Gilewski et al., 2016). Tensegrity plate-like structures can also be built with tensegrity modules, such as a simplex or an expanded octahedron. Even very simple tensegrity structures have complex geometry and unique features. Their structural behaviour can be explained using a continuum model of a three-dimensional tensegrity plate-like structure (Al Sabouni-Zawadzka et al., 2016).

In the paper, a continuum model of the plate is used. The model includes the effect of self-stress initially applied to the tensegrity structure. In the analysis, a linear six-parameter shell theory (Chróścielewski et al., 2004; Pietraszkiewicz, 2016) is proposed. In considerations, the shell theory is simplified by assuming that the plates have no curvature. As a result, the two-dimensional plate model for moderately thick plates is obtained for both membrane and bending deformations. Additionally, different planes of support of tensegrity plate-like structures are taking into account, thus different reference planes of the plate model - the lower surface, the middle surface and the upper surface are considered. The proposed approach allows one to analyse the influence of self-stress states and some geometrical parameters on average displacements, strains and internal forces in the structures. 
The model used in the paper is valid for all structures composed of tensegrity modules with orthotropic properties.

\section{Material and methods}

\subsection{Linear six-parameter shell theory for the orthotropic model}

Developments of the theory of elastic shells have been widely discussed in papers and monographs. In the paper, the six-parameter (six-field) shell theory is used (Burzyński et al., 2016; Chróścielewski et al., 2004, 2011, 2016; Pietraszkiewicz, 2016; Witkowski, 2011). This kinematic model is formally equivalent to the Cosserat continuum with six independent degrees of freedom: three translations and three rotations (with the drilling degree of freedom). It is assumed that translations and rotations are small, i.e., the linear six-parameter shell theory can be used. As one of the first, the linear constitutive equation for Cosserat continuum was derived by Nowacki (1971). In this approach, the linear six-parameter shell theory is applied to the analysis of tensegrity plate-like structures.

The rectangular plate of a constant thickness $h$ in the Cartesian coordinate system $\left(x_{1}, x_{2}, z\right)$ is considered (Fig. 1). According to the Hencky-Boole kinematic hypothesis, a displacement field in the $3 \mathrm{D}$ space is described as

$$
\widetilde{\mathbf{u}}\left(x_{\alpha}, z\right)=\mathbf{u}\left(x_{\alpha}\right)+z \boldsymbol{\beta}\left(x_{\alpha}\right) \quad \text { for } \quad \alpha=1,2
$$

where $\mathbf{u}$ is the translation vector and $\boldsymbol{\beta}$ is the rotation vector of the reference surface (2D)

$$
\mathbf{u}\left(x_{\alpha}\right)=\left[u_{\alpha}, w\right]^{\mathrm{T}} \quad \boldsymbol{\beta}\left(x_{\alpha}\right)=\left[\phi_{\alpha}, \psi\right]^{\mathrm{T}}
$$

The proposed approach, compared to the classical five-parameter theory, includes additionally third independent non-vanishing rotation - the drilling degree of freedom $\psi$ (rotation about the normal to the surface).
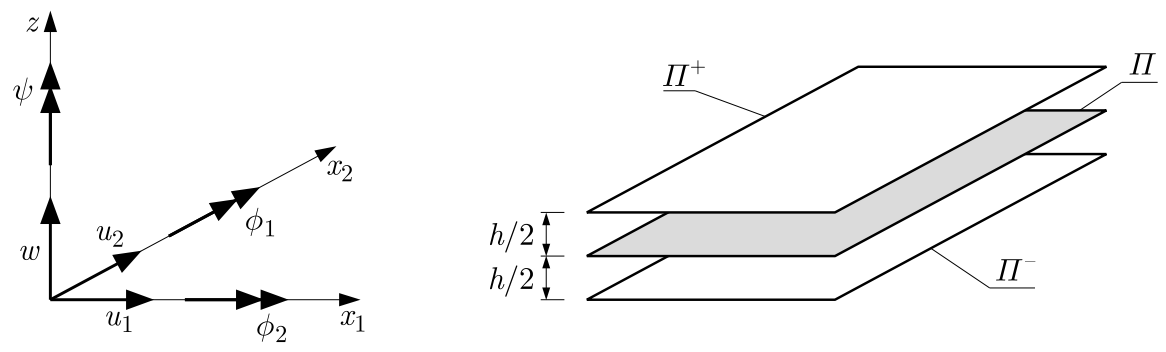

Fig. 1. Geometry of a 3D plate-like body

In a plate, there is no curvature, wchich means that the curvature of tensors is equal to zero, consequently, a model analysed in the paper is simpler than that shown by Chróścielewski et al. (2004) and by Pietraszkiewicz (2016). Consequently, the linear six-parameter shell theory is simplified. Below the reduced relations and equations are showed:

— the kinematic relations

$$
\gamma_{\alpha \beta}=u_{\alpha, \beta}-\epsilon_{\alpha \beta} \psi \quad \gamma_{\alpha 3}=\phi_{\alpha}+w_{, \alpha} \quad \kappa_{\alpha \beta}=\phi_{\alpha, \beta} \quad \kappa_{\alpha 3}=\psi_{, \alpha}
$$

where $\gamma_{\alpha \beta}, \gamma_{\alpha 3}, \kappa_{\alpha \beta}, \kappa_{\alpha 3}$ are the strain components and $\epsilon_{\alpha \beta}$ is the Ricci symbol, - the internal forces

$$
\begin{array}{ll}
N_{\alpha \beta}=\int_{z_{1}}^{z_{2}} S_{\alpha \beta} d z & N_{\alpha 3}=\int_{z_{1}}^{z_{2}} S_{\alpha 3} d z \\
M_{\alpha \beta}=\int_{z_{1}}^{z_{2}} S_{\alpha \beta} z d z & M_{\alpha 3}=\int_{z_{1}}^{z_{2}} S_{\alpha 3} z d z
\end{array}
$$


where $S_{\alpha \beta}$ and $S_{\alpha 3}$ are the stress components,

— the equilibrium equations

$$
\begin{array}{ll}
N_{\alpha \beta, \alpha}+f_{\beta}=0 & N_{\alpha 3, \alpha}+f_{3}=0 \\
M_{\alpha \beta, \alpha}+N_{\beta 3}+m_{\beta}=0 & M_{\alpha 3, \alpha}+\epsilon_{\alpha \beta} N_{\alpha \beta}+m_{3}=0
\end{array}
$$

where $f_{\beta}, f_{3}, m_{\beta}, m_{3}$ are the external loads.

Internal forces (2.4) depend on the reference surface. In the paper, three different reference surfaces of the plate model are considered: the lower $\Omega^{L}$, the middle $\Omega^{M}$ and the upper $\Omega^{U}$. In the mentioned approaches, the domains of the plate are defined as follows (Fig. 1)

$$
\begin{aligned}
& \Omega^{L}=\left\{x_{K}: \quad x_{\alpha} \in \Pi^{-}, z \in\left\langle z_{1}, z_{2}\right\rangle ; z_{1}=0, z_{2}=h\right\} \\
& \Omega^{M}=\left\{x_{K}: \quad x_{\alpha} \in \Pi, z \in\left\langle z_{1}, z_{2}\right\rangle ; z_{1}=-h / 2, z_{2}=h / 2\right\} \\
& \Omega^{U}=\left\{x_{K}: \quad x_{\alpha} \in \Pi^{+}, z \in\left\langle z_{1}, z_{2}\right\rangle ; z_{1}=-h, z_{2}=0\right\}
\end{aligned}
$$

Consequently, limitation of integrations (2.4) for each surface follows from the domains definitions.

A complete six-parameter linear shell theory containing the drilling rotation $\psi$, two workconjugate drilling bending measures $\kappa_{\alpha 3}$ and two drilling couples $M_{\alpha 3}$ is presented in the paper. The constitutive equation for the linear theory of elasticity is expressed as

$$
S_{i j}=D_{i j k l} E_{k l} \quad i, j, k, l=1,2,3
$$

where $S_{i j}$ is the component of the stress tensor, $E_{k l}$ is the component of the strain tensor and $D_{i j k l}$ is the component of the fourth-rank tensor of elasticity. In general, a tensor of elasticity contains 36 independent components, but taking into account the symmetry of strain energy 21 distinct components can be set out. The number of independent components is further reduced if the material has symmetry planes. There are exactly eight different sets of symmetry planes (Chadwick et al., 2001). One of them is orthogonal symmetry (orthotropic material). This material requires 9 elastic constants in a two-dimensional case.

In the paper, the orthotropic tensegrity plate-like structures based on the Reissner-Mindlin theory are discussed. For this model, the tensor of elasticity can be written as a matrix $\left\{D_{i j k l}\right\} \equiv \mathbf{d}$ which contains 6 nonzero independent components

$$
\begin{array}{lll}
B_{1111}^{0}=d_{11} & B_{2222}^{0}=d_{22} & 2 B_{2323}^{0}=d_{44} \\
2 B_{1313}^{0}=d_{55} & 2 B_{1212}^{0}=d_{66} & B_{1122}^{0}=d_{12}
\end{array}
$$

The stress tensor and the strain tensor are written as

$$
S_{i j}=\left\{\begin{array}{lllll}
S_{11} & S_{22} & S_{23} & S_{13} & S_{12}
\end{array}\right\}^{\mathrm{T}} \quad E_{k l}=\left\{\begin{array}{lllll}
E_{11} & E_{22} & 2 E_{23} & 2 E_{13} & 2 E_{12}
\end{array}\right\}^{\mathrm{T}}
$$

where

$$
\begin{aligned}
& E_{11}=\gamma_{11}+z \kappa_{11} \quad E_{22}=\gamma_{22}+z \kappa_{22} \quad E_{23}=\frac{1}{2}\left(\gamma_{23}+z \kappa_{23}\right. \\
& E_{13}=\frac{1}{2}\left(\gamma_{13}+z \kappa_{13}\right) \quad E_{12}=\frac{1}{2}\left(\gamma_{12}+\gamma_{21}+z \kappa_{12}+z \kappa_{21}\right)
\end{aligned}
$$

Stress components $(2.9)_{1}$ are received from constitutive equations (2.7). Next, taking into account the lower, middle and upper reference surfaces, the internal forces (2.4) are calculated

$$
\begin{aligned}
& N_{11}=h_{0} d_{11} \gamma_{11}+h_{0} d_{12} \gamma_{22}+h_{1} d_{11} \kappa_{11}+h_{1} d_{12} \kappa_{22} \\
& N_{22}=h_{0} d_{12} \gamma_{11}+h_{0} d_{22} \gamma_{22}+h_{1} d_{12} \kappa_{11}+h_{1} d_{22} \kappa_{22} \\
& N_{12}=h_{0} d_{66}\left(\gamma_{12}+\gamma_{21}\right)+h_{1} d_{66}\left(\kappa_{12}+\kappa_{21}\right) \\
& N_{13}=\alpha_{0} h_{0} d_{55} \gamma_{13}+\alpha_{1} h_{1} d_{55} \kappa_{13} \quad N_{23}=\alpha_{0} h_{0} d_{44} \gamma_{23}+\alpha_{1} h_{1} d_{44} \kappa_{23}
\end{aligned}
$$


and

$$
\begin{aligned}
& M_{11}=h_{1} d_{11} \gamma_{11}+h_{1} d_{12} \gamma_{22}+h_{2} d_{11} \kappa_{11}+h_{2} d_{12} \kappa_{22} \\
& M_{22}=h_{1} d_{12} \gamma_{11}+h_{1} d_{22} \gamma_{22}+h_{2} d_{12} \kappa_{11}+h_{2} d_{22} \kappa_{22} \\
& M_{12}=h_{1} d_{66}\left(\gamma_{12}+\gamma_{21}\right)+h_{2} d_{66}\left(\kappa_{12}+\kappa_{21}\right) \\
& M_{13}=\alpha_{1} h_{1} d_{55} \gamma_{13}+\alpha_{2} h_{2} d_{55} \kappa_{13} \quad M_{23}=\alpha_{1} h_{1} d_{44} \gamma_{23}+\alpha_{2} h_{2} d_{44} \kappa_{23}
\end{aligned}
$$

where $h_{i}(i=0,1,2)$ are parameters depending on the reference surfaces (values are shown in Table 1$)$ and $\alpha_{i}(i=0,1,2)$ are shear correction factors.

The problem of determining the shear factor was created in the Timoshenko beam theory, which takes into account the transverse shear deformation and the rotatory inertia (Khorshidi and Shariati, 2017; Obara and Gilewski, 2016; Timoshenko and Gere, 1961). Within the general six-parameter shell model used here, the shear correction factors $\alpha_{0}$ and $\alpha_{2}$ are introduced into the constitutive equations for the respective transverse shear stress resultants and stress couples (Chróścielewski et al., 1997). The values of two correction factors $\alpha_{0}=5 / 6$ and $\alpha_{2}=7 / 10$, with detailed derivation of these values, were arrived by Pietraszkiewicz (1979). The more information about the shear factors and the influence of different values of these factors on the results of static and dynamic behaviour of shell structures can be found in Chróścielewski et al. (2000). In the paper, additionally a correction factor $\alpha_{1}$ is introduced. This factor is significant if the lower surface or the upper surface is used as the reference plane.

The closed form of the equilibrium equations for a plate $\left(x_{1} \in\langle 0, a\rangle\right.$ and $\left.x_{2} \in\langle 0, b\rangle\right)$ and a plate strip $\left(x_{1} \in\langle 0, a\rangle\right.$ and $\left.x_{2} \in(-\infty,+\infty)\right)$ can be obtained by inserting Eqs. (2.11) and (2.12) into Eq. (2.5).

\subsubsection{Plate}

The behaviour at any point of the considered plate is defined by generalized displacements $\mathbf{q}$ and the corresponding to them internal forces $\mathbf{Q}$

$$
\begin{aligned}
& \mathbf{q}=\mathbf{q}\left(x_{1}, x_{2}\right)=\left[u_{1}, u_{2}, \psi, \phi_{1}, \phi_{2}, w\right]^{\mathrm{T}} \\
& \mathbf{Q}=\mathbf{Q}\left(x_{1}, x_{2}\right)=-\left[f_{1}, f_{2}, m_{3}, m_{1}, m_{2}, f_{3}\right]^{\mathrm{T}}
\end{aligned}
$$

The first three displacements in $(2.13)_{1}$ describe the membrane state and the last three - the bending state. In general, these states are coupled. Equilibrium equations (2.5) for the plate can be written as

$$
\mathbf{L q}=\mathbf{Q}
$$

where

$$
\mathbf{L}=\left[\begin{array}{cccccc}
h_{0} L_{1} & h_{0} L_{4} & 0 & h_{1} L_{1} & h_{1} L_{4} & 0 \\
h_{0} L_{4} & h_{0} L_{2} & 0 & h_{1} L_{4} & h_{1} L_{2} & 0 \\
0 & 0 & \alpha_{2} h_{2} L_{3} & \alpha_{1} h_{1} L_{5} & \alpha_{1} h_{1} L_{6} & \alpha_{1} h_{1} L_{3} \\
h_{1} L_{1} & h_{1} L_{4} & -\alpha_{1} h_{1} L_{5} & h_{2} L_{1}-\alpha_{0} h_{0} d_{55} & h_{2} L_{4} & -\alpha_{0} h_{0} L_{5} \\
h_{1} L_{4} & h_{1} L_{2} & -\alpha_{1} h_{1} L_{6} & h_{2} L_{4} & h_{2} L_{2}-\alpha_{0} h_{0} d_{44} & -\alpha_{0} h_{0} L_{6} \\
0 & 0 & \alpha_{1} h_{1} L_{3} & \alpha_{0} h_{0} L_{5} & \alpha_{0} h_{0} L_{6} & \alpha_{0} h_{0} L_{3}
\end{array}\right]
$$

where

$$
\begin{aligned}
& L_{1}=d_{11} \frac{\partial^{2}}{\partial x_{1}^{2}}+d_{66} \frac{\partial^{2}}{\partial x_{2}^{2}} \\
& L_{2}=d_{66} \frac{\partial^{2}}{\partial x_{1}^{2}}+d_{22} \frac{\partial^{2}}{\partial x_{2}^{2}} \\
& L_{4}=\left(d_{12}+d_{66}\right) \frac{\partial^{2}}{\partial x_{1} \partial x_{2}} \\
& L_{5}=d_{55} \frac{\partial}{\partial x_{1}} \\
& L_{3}=d_{55} \frac{\partial^{2}}{\partial x_{1}^{2}}+d_{44} \frac{\partial^{2}}{\partial x_{2}^{2}} \\
& L_{6}=d_{44} \frac{\partial}{\partial x_{2}}
\end{aligned}
$$




\subsubsection{Plate strip}

The behaviour of the considered plate strip, with width $a$, is defined by generalized displacements $\widetilde{\mathbf{q}}$ and the corresponding to them internal forces $\widetilde{\mathbf{Q}}$

$$
\widetilde{\mathbf{q}}=\widetilde{\mathbf{q}}\left(x_{1}\right)=\left[u_{1}, \psi, \phi_{1}, w\right]^{\mathrm{T}} \quad \widetilde{\mathbf{Q}}=\widetilde{\mathbf{Q}}\left(x_{1}\right)=-a^{2}\left[f_{1}, m_{3}, m_{1}, f_{3}\right]^{\mathrm{T}}
$$

Equilibrium equations (2.5) for the plate strip can be written as

$$
\widetilde{\mathbf{L}} \widetilde{\mathbf{q}}=\widetilde{\mathbf{Q}}
$$

where

$$
\widetilde{\mathbf{L}}=\left[\begin{array}{cccc}
A_{0} \widetilde{L}_{1} & 0 & A_{1} \widetilde{L}_{1} & 0 \\
0 & B_{2} \widetilde{L}_{1} & a B_{1} \widetilde{L}_{2} & B_{2} \widetilde{L}_{1} \\
A_{1} \widetilde{L}_{1} & -a B_{1} \widetilde{L}_{2} & -a^{2} B_{0}+A_{2} \widetilde{L}_{1} & -a B_{0} \widetilde{L}_{2} \\
0 & B_{1} \widetilde{L}_{1} & a B_{0} \widetilde{L}_{2} & B_{0} \widetilde{L}_{1}
\end{array}\right]
$$

where

$$
\begin{aligned}
\widetilde{L}_{1}=\frac{d^{2}}{d \xi^{2}} & \widetilde{L}_{2}=\frac{d}{d \xi} & \xi=\frac{x_{1}}{a} & \\
A_{i}=h_{i} d_{11} & B_{i}=\alpha_{i} h_{i} d_{55} & \text { for } & i=0,1,2
\end{aligned}
$$

The parameters $A_{i}$ and $B_{i}$ depend on the reference surfaces. The formulas of these parameters are shown in Table 1. For the plate strip, as in the case of the plate, the membrane state and the bending state are coupled.

Table 1. Formulas of the parameters $h_{i}, A_{i}$ and $B_{i}$ for the reference surfaces

\begin{tabular}{|c|c|c|c|}
\hline Parameters & Lower surface & Middle surface & Upper surface \\
\hline \hline$h_{0}$ & $h$ & $h$ & $h$ \\
\hline$h_{1}$ & $h^{2} / 2$ & 0 & $-h^{2} / 2$ \\
\hline$h_{2}$ & $h^{3} / 3$ & $h^{3} / 12$ & $h^{3} / 3$ \\
\hline$A_{0}$ & $h d_{11}$ & $h d_{11}$ & $h d_{11}$ \\
\hline$A_{1}$ & $h^{2} d_{11} / 2$ & 0 & $-h^{2} d_{11} / 2$ \\
\hline$A_{2}$ & $h^{3} d_{11} / 3$ & $h^{3} d_{11} / 12$ & $h^{3} d_{11} / 3$ \\
\hline$B_{0}$ & $\alpha_{0} h d_{55}$ & $\alpha_{0} h d_{55}$ & $\alpha_{0} h d_{55}$ \\
\hline$B_{1}$ & $\alpha_{1} h^{2} d_{55} / 2$ & 0 & $-\alpha_{1} h^{2} d_{55} / 2$ \\
\hline$B_{2}$ & $\alpha_{2} h^{3} d_{55} / 3$ & $\alpha_{2} h^{3} d_{55} / 12$ & $\alpha_{2} h^{3} d_{55} / 3$ \\
\hline
\end{tabular}

Solving the set of differential equations (2.18) it is possible to obtain explicit formulas of the displacement and internal force. The mentioned formulas are described as follows

$$
\begin{aligned}
u_{1}(\xi) & =C_{5}+C_{6} \xi+\frac{3 A_{1}}{a A_{0}} C_{4} \xi^{2}-\frac{a^{2}}{2 A_{0}} f_{1} \xi^{2}+\frac{a^{3} E_{1}}{6} f_{3} \xi^{3} \\
\psi(\xi) & =C_{7}+C_{8} \xi+\frac{a^{2}}{2}\left(D_{1} f_{3}-D_{0} m_{3}\right) \xi^{2} \\
\phi_{1}(\xi) & =-\frac{1}{a}\left(C_{2}+2 C_{3} \xi+3 C_{4} \xi^{2}+\frac{6}{a^{2} B_{0} E_{0}} C_{4}+\frac{B_{1}}{B_{0}} C_{8}\right)-\frac{a}{B_{0}}\left(1+B_{1} D_{1}\right) f_{3} \xi \\
- & \frac{a^{3} E_{0}}{6} f_{3} \xi^{3}+a D_{1} m_{3} \xi+\frac{1}{B_{0}} m_{1}-\frac{A_{1}}{A_{0} B_{0}} f_{1} \\
w(\xi) & =C_{1}+C_{2} \xi+C_{3} \xi^{2}+C_{4} \xi^{3}+\frac{a^{4} E_{0}}{24} f_{3} \xi^{4}
\end{aligned}
$$


and

$$
\begin{aligned}
& N_{11}(\xi)=-\frac{2 A_{1}}{a^{2}} C_{3}+\frac{A_{0}}{a} C_{6}-a f_{1} \xi-\frac{A_{1}}{B_{0}}\left(1+B_{1} D_{1}\right) f_{3}+A_{1} D_{1} m_{3} \\
& N_{13}(\xi)=-\frac{6}{a^{3} E_{0}} C_{4}-a f_{3} \xi-\frac{A_{1}}{A_{0}} f_{1}+m_{1} \\
& M_{11}(\xi)=-\frac{2 A_{2}}{a^{2}} C_{3}-\frac{6}{a^{2} E_{0}} C_{4} \xi+\frac{A_{1}}{a} C_{6}-\frac{a A_{1}}{A_{0}} f_{1} \xi+\frac{a^{2}}{2}\left(A_{1} E_{1}-A_{2} E_{0}\right) f_{3} \xi^{2} \\
& -\frac{A_{2}}{B_{0}}\left(1+B_{1} D_{1}\right) f_{3}+A_{2} D_{1} m_{3} \\
& M_{13}(\xi)=-\frac{6 B_{1}}{a^{3} B_{0} E_{0}} C_{4}+\frac{1}{a D_{0}} C_{8}+a\left(B_{1} D_{1}-B_{2} D_{2}\right) m_{3} \xi-\frac{A_{1} B_{1}}{A_{0} B_{0}} f_{1}+\frac{B_{1} m_{1}}{B_{0}}
\end{aligned}
$$

where

$$
D_{i}=\frac{B_{i}}{B_{0} B_{2}-B_{1}^{2}} \quad E_{i}=\frac{A_{i}}{A_{0} A_{2}-A_{1}^{2}} \quad \text { for } \quad i=0,1,2
$$

The results for the plate and plate strip can be used in analysis of different types of orthotropic systems, such as beams, plate strips, plates or more complicated multi-module plate-like structures. The displacement and internal force functions of these systems depend on the components of elastic matrix (2.8).

To illustrate the proposed approach, a continuum orthotropic model of the tensegrity plate-like structure is used. The internal and external plane of support of the structure is taken into account. For tensegrity systems, the components of the elastic matrix depend on stiffness of cables and struts and on the level of self-stress.

\subsection{Orthotropic tensegrity plate-like structure}

The orthotropic model of a tensegrity plate-like structure is based on the energetic equivalence between the discrete tensegrity repeatable element and the continuum model of the orthotropic material element shown in Fig. 2 (Al Sabouni-Zawadzka and Gilewski, 2016; Kebiche et al., 2008). This approach can be used to a chosen structure of repeatable tensegrity elements creating plate-like structures.
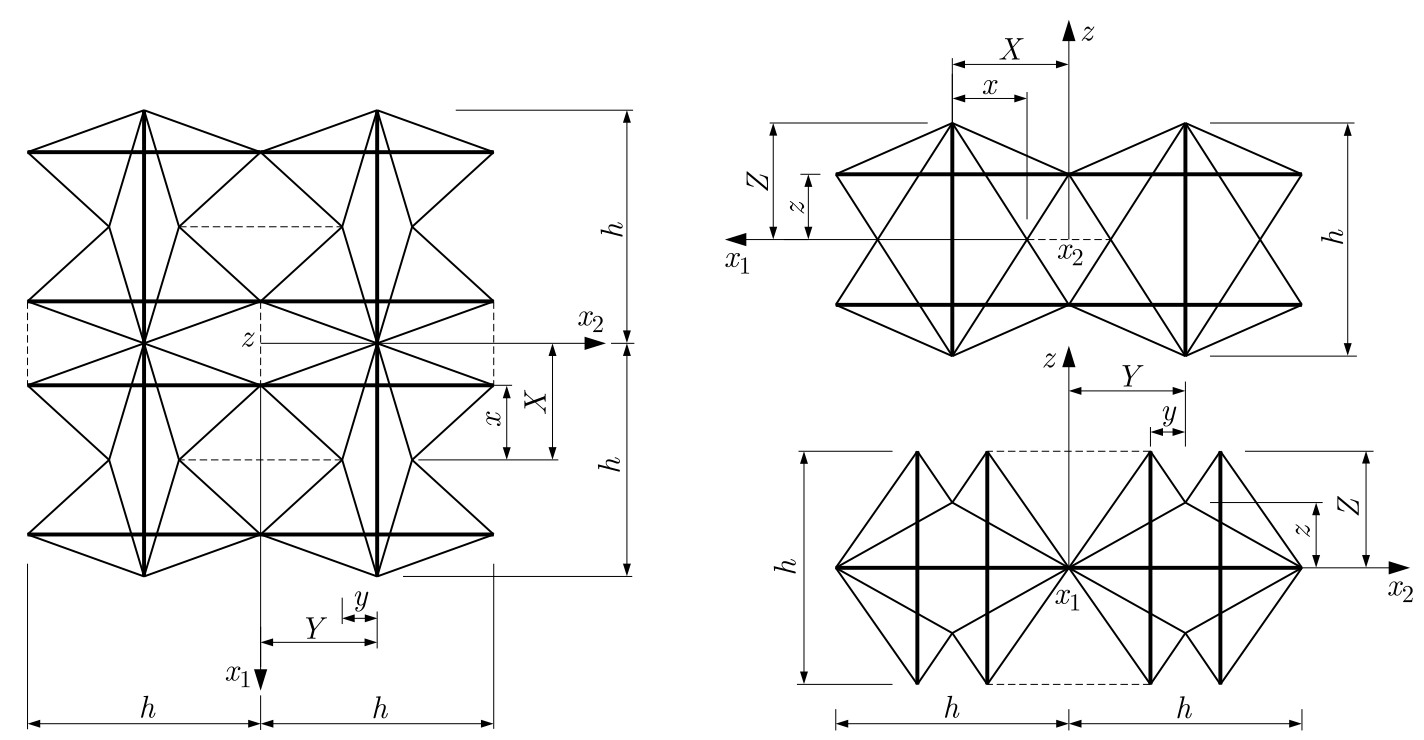

Fig. 2. Views of expanded four-strut octahedron modules with additional cables (doted line) 
A system of fully connected, repeating expanded four-strut octahedron modules with additional cables (Al Sabouni-Zawadzka et al., 2016) is used as an example. The system is orthotropic for the following geometric parameters $x / X=0.65, y / Y=0.3$ and $z / Z=0.56$. This system consists of struts, regular cables and connecting cables, which are described by the following coefficients

$$
n=\frac{(E A)_{\text {cable }}}{(E A)_{\text {struct }}} \quad m=\frac{(E A)_{\text {conection }}}{(E A)_{\text {struct }}} \quad \sigma=\frac{S}{(E A)_{\text {struct }}}
$$

where $E$ is the Young modulus, $A$ is cross section area and $S$ is the axial force. The coefficients $n$ and $m$ describe the proportions of member properties, and $\sigma$ describes the level of self-stress in tensegrity structures.

The self-stress state is the most important feature of tensegrity structures. This state makes the structure as strong as the self-supporting structure and stabilizes the infinitesimal mechanisms occurred in tensegrity structures. The impact of the self-stress state in the structure is taken into account by using the geometric stiffness matrix.

The coefficients of elastic matrix (2.8) of the tensegrity plate-like structure are as follows:

$$
\begin{aligned}
& d_{11}=\frac{2 E A}{h^{2}} \delta_{11} \quad d_{22}=\frac{2 E A}{h^{2}} \delta_{22} \quad d_{12}=\frac{E A}{h^{2}} \delta_{12} \\
& d_{44}=\frac{E A}{h^{2}} \delta_{23} \quad d_{55}=\frac{E A}{h^{2}} \delta_{13} \quad d_{66}=\frac{E A}{h^{2}} \delta_{12}
\end{aligned}
$$

where

$$
\begin{array}{ll}
E A=(E A)_{\text {struct }} & \delta_{12}=0.845615 n-0.105243 \sigma \\
\delta_{11}=1+1.52325 n+0.13125 m+0.129225 \sigma & \delta_{13}=1.26604 n-0.153207 \sigma \\
\delta_{22}=1+1.35912 n+0.35 m+0.137028 \sigma & \delta_{23}=1.51283 n-0.168813 \sigma
\end{array}
$$

\section{Results and discussion}

Displacements and the internal forces for tensegrity plate-like structures, as a function of parameters (2.26), and, in consequence, coefficients (2.24) are determined. The jointly supported rectangular plate and plate strips with different kinds of boundary conditions are discussed. The different planes of support in tensegrity plate-like structures are analysed. The results of the analysis are described using $L$ for the lower surface, $M$ for the middle surface and $U$ for the upper surface.

The displacements and internal forces are presented in the closed form. Displacements as a function of coefficients $n$ and $\sigma$ are presented in the form of graphs. Constants $C_{i}(i=P, F, M)$ are not taken into account in the graphs.

\subsection{Jointly supported rectangular plate}

The first example is the jointly supported plate with a sinusoidal load (Fig. 3)

$$
f_{3}\left(x_{1}, x_{2}\right)=-q_{0} \sin \frac{\pi x_{1}}{a} \cos \frac{\pi x_{2}}{b}
$$

Solving equation (2.14) by applying the Fourier sine and cosine series that satisfy the boundary conditions, leads to determining the displacements for the lower, the middle and the upper surface. The closed form of solutions for the maximum deflection $w_{\max }=w(0.5 a, 0.5 b)$ for a square plate $(b=a)$ are as follows 


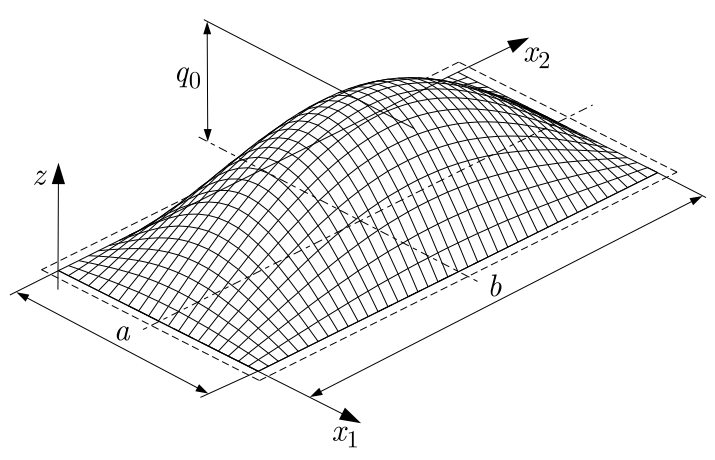

Fig. 3. Rectangular plate jointly supported

$$
\begin{aligned}
& w_{\text {max }}^{M}=C_{P}\left(\frac{144 \alpha_{0}^{2} a^{4} \beta_{0}+h^{2} \pi^{2} \beta_{2}+12 \alpha_{0} a^{2} h^{2} \pi^{2} \beta_{3}}{\alpha_{0}\left(\beta_{1} \beta_{2}+\beta_{4}\right)}\right) \\
& w_{\text {max }}^{L}=w_{\text {max }}^{U}=C_{P}\left(\frac{4\left(36 \alpha_{0} \alpha_{3} a^{4} \beta_{0}+\alpha_{2} h^{2} \pi^{2} \beta_{2}\right) \beta_{1}+12 a^{2} h^{2} \pi^{2}\left(4 \alpha_{0} \alpha_{2} \beta_{1} \beta_{3}-\beta_{5}\right)}{\alpha_{3}\left(\beta_{1} \beta_{2}+\beta_{4}\right) \beta_{1}}\right)
\end{aligned}
$$

where

$$
\begin{aligned}
& C_{P}=\frac{a^{2} q_{0}}{E A h \pi^{4}} \quad \beta_{0}=\delta_{13} \delta_{23} \quad \beta_{1}=\delta_{13}+\delta_{23} \\
& \beta_{2}=h^{2} \pi^{2}\left[\delta_{12}\left(2 \delta_{22}-3 \delta_{12}\right)+2 \delta_{11}\left(\delta_{12}+2 \delta_{22}\right)\right] \\
& \beta_{3}=\delta_{13}\left(\delta_{12}+2 \delta_{22}\right)+\delta_{23}\left(\delta_{12}+2 \delta_{11}\right) \quad \beta_{4}=24 \alpha_{0} a^{2} \delta_{13} \delta_{23}\left(\delta_{11}+\delta_{22}+3 \delta_{12}\right) \\
& \beta_{5}=3 \alpha_{1}^{2}\left[\delta_{13}^{2}\left(\delta_{12}+2 \delta_{22}\right)+\delta_{23}^{2}\left(\delta_{12}+2 \delta_{11}\right)-4 \delta_{12} \delta_{13} \delta_{23}\right]
\end{aligned}
$$

For any rectangular plate, the decoupling of bending and membrane behaviour occurs only for the middle reference surface. It means that for this surface the displacements describing the membrane state are equal to zero: $u_{1}^{M}(\xi)=u_{2}^{M}(\xi)=\psi^{M}(\xi)=0$. For the lower and the upper surface, the vertical displacements are the same $(3.2)_{2}$, and the membrane displacements are as follows: $u_{1}^{L}(\xi)=-u_{1}^{U}(\xi), u_{2}^{L}(\xi)=-u_{2}^{U}(\xi)$ and $\psi^{L}(\xi)=-\psi^{U}(\xi)$. The rotations $\phi_{\alpha}(\xi)$ do not depend on the reference surface: $\phi_{1}^{L}(\xi)=\phi_{1}^{M}(\xi)=\phi_{1}^{U}(\xi)$ and $\phi_{2}^{L}(\xi)=\phi_{2}^{M}(\xi)=\phi_{2}^{U}(\xi)$. For the analysed plate, the displacements depend on three shear correction factors $\alpha_{i}(i=0,1,2)$ and on the parameter

$$
\alpha_{3}=4 \alpha_{0} \alpha_{2}-3 \alpha_{1}^{2}
$$

However, if the middle reference surface is considered only one shear factor $\alpha_{0}$ occurs.

The paper refers to the application of the linear six-parameter shell theory to the analysis of orthotropic tensegrity plate-like structures. These systems are double-layer tensegrity grids. To establish values of the shear correction factors for such a plate, it is necessary to build and next to verify the proper model which should be based on the knowledge of the modelled material and the phenomenon. The aim of future research will establish values of the three shear correction factors within the six-parameter linear theory of elastic tensegrity plate-like structures and test their influence on numerical results of static behavior of such structures.

Now, to illustrate the influence of the reference plane on the behaviour of tensegrity structures, it is that the assumed values of the shear factors are: $\alpha_{0}=5 / 6, \alpha_{2}=7 / 10$ (Chróścielewski et al., 1997, 2000; Pietraszkiewicz, 1979; Witkowski, 2011; Woźniak, 2001) and $\alpha_{1}=8 / 10$. The last correction factor has been assumed so that parameter (3.4) is positive definite.

On the basis of Eq. (3.2), the influence of the self-stress and stiffness of cables and struts on the displacement in tensegrity plate-like structures can be estimated in a simple way. The parametric analysis can be carried for any moderately thick plates. As an example, results for the thickness to length ratio $h / a=0.25$, on the assumption $m=n$, are shown in Fig. 4. Formulas (3.2) are valid only for $n>0.2 \sigma$. 

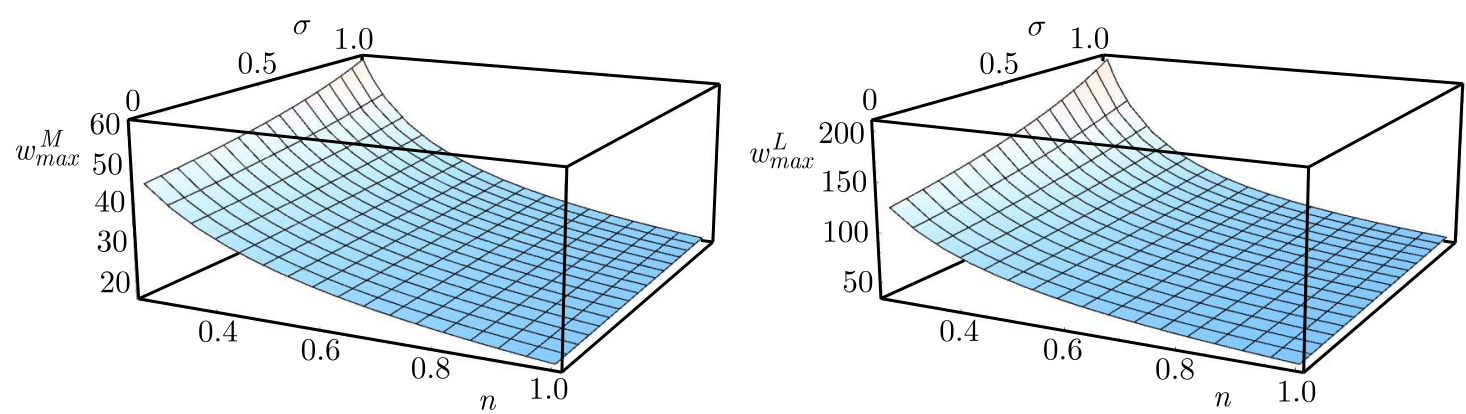

Fig. 4. Maximum deflection of the plate

\subsection{Plate strips}

The displacements and the internal forces for plate strips are determined based on Eqs. (2.21) and (2.22). These formulas depend on boundary conditions. Plate strips with the force mass $f_{3}\left(x_{1}\right)=-q_{0}$ taken into account are studied. Three kinds of support, i.e., cantilever (Fig. 5a), simply supported (Fig. 5b) and clamped-clamped (Fig. 5c) are considered. The formulas of the maximum deflection are derived:

— the cantilever plate strip

$$
w_{\max }^{M}=C_{M}\left[6 \frac{1}{\delta_{11}}+\frac{4}{\alpha_{0}}\left(\frac{h}{a}\right)^{2} \frac{1}{\delta_{13}}\right] \quad w_{\max }^{L}=w_{\max }^{U}=C_{M}\left[6 \frac{1}{\delta_{11}}+16 \frac{\alpha_{2}}{\alpha_{3}}\left(\frac{h}{a}\right)^{2} \frac{1}{\delta_{13}}\right]
$$

— the simply supported plate strip

$$
w_{\max }^{M}=C_{M}\left[\frac{5}{8} \frac{1}{\delta_{11}}+\frac{1}{\alpha_{0}}\left(\frac{h}{a}\right)^{2} \frac{1}{\delta_{13}}\right] \quad w_{\max }^{L}=w_{\max }^{U}=C_{M}\left[\frac{1}{4} \frac{1}{\delta_{11}}+4 \frac{\alpha_{2}}{\alpha_{3}}\left(\frac{h}{a}\right)^{2} \frac{1}{\delta_{13}}\right]
$$

- the clamped-clamped plate strip

$$
w_{\max }^{M}=C_{M}\left[\frac{1}{8} \frac{1}{\delta_{11}}+\frac{1}{\alpha_{0}}\left(\frac{h}{a}\right)^{2} \frac{1}{\delta_{13}}\right] \quad w_{\max }^{L}=w_{\max }^{U}=C_{M}\left[\frac{1}{8} \frac{1}{\delta_{11}}+4 \frac{\alpha_{2}}{\alpha_{3}}\left(\frac{h}{a}\right)^{2} \frac{1}{\delta_{13}}\right]
$$

where $C_{M}=-a^{4} q_{0} /(8 E A h)$. In these cases, the deflection functions depend on the correction factors $\alpha_{i}(i=0,1,2,3)$ but for the middle references surface only on the factor $\alpha_{0}$.

(a)

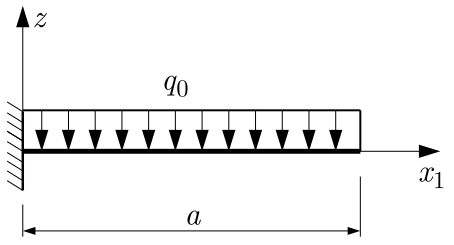

(b)

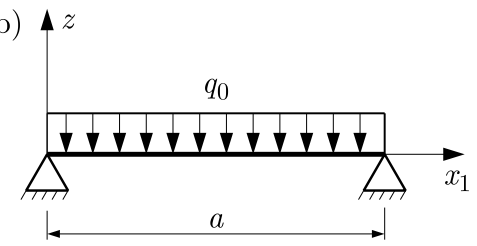

(c)

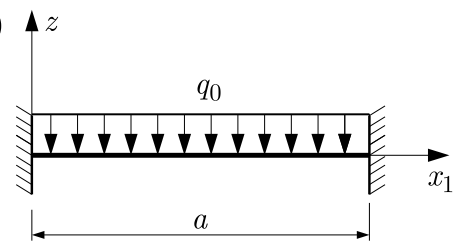

Fig. 5. Plate strips: (a) cantilever, (b) simply supported, (c) clamped-clamped

For all analysed plate strips, displacements describing the membrane state are equal to zero only for the middle reference surface: $u_{1}^{M}(\xi)=\psi^{M}(\xi)=0$. For the lower and the upper surfaces, the axial displacement is a polynomial function of the degree three, and for each surface there is an equality: $u_{1}^{L}(\xi)=-u_{1}^{U}(\xi)$. The drilling rotation is a polynomial function of the degree two and $\psi^{L}(\xi)=-\psi^{U}(\xi)$. The rotations $\phi_{\alpha}(\xi)$ do not depend on the reference surface, and for each surface: $\phi_{1}^{L}(\xi)=\phi_{1}^{M}(\xi)=\phi_{1}^{U}(\xi)$ and $\phi_{2}^{L}(\xi)=\phi_{2}^{M}(\xi)=\phi_{2}^{U}(\xi)$.

For the cantilever and the clamped-clamped plate strip, the internal forces do not depend on the reference surface. The membrane force and the drilling couple are equal to zero: $N_{11}(\xi)=0$, $M_{13}(\xi)=0$, and the transverse force and the bending couple are as follows: 
— the cantilever plate strip

$$
N_{13}(\xi)=-a q_{0}(1-\xi) \quad M_{11}(\xi)=-\frac{a^{2} q_{0}}{2}\left(1+2 \xi-\xi^{2}\right)
$$

— the clamped-clamped plate strip

$$
N_{13}(\xi)=-\frac{a q_{0}}{2}(1-2 \xi) \quad M_{11}(\xi)=\frac{a^{2} q_{0}}{12}\left(1-6 \xi+6 \xi^{2}\right)
$$

For the simply supported plate strip, the transverse force and the bending couple do not depend on the reference surface, as for previous cases

$$
N_{13}(\xi)=-\frac{a q_{0}}{2}(1-2 \xi) \quad M_{11}(\xi)=-\frac{a^{2} q_{0}}{2}\left(\xi-\xi^{2}\right)
$$

the drilling couple is equal to zero $M_{13}(\xi)=0$, but for the lower and the upper surface, the constant axial force occurs additionally

$$
N_{11}^{L}(\xi)=-N_{11}^{U}(\xi)=-\frac{a^{2} q_{0}}{8 h} \quad N_{11}^{M}(\xi)=0
$$

Internal forces (3.8)-(3.11) do not depend on geometric and physical properties of tensegrity plate-like structures. Based on Eqs. (3.5)-(3.7), the influence of the self-stress and the stiffness of cables and struts on the displacement in tensegrity plate-like structures can be estimated. As an example, the parametric results, for $h / a=0.25$, on the assumption $m=n$, are represented graphically in Figs. 6-8. Values of the correction factors are assumed like for the analysed jointly supported plate. Formulas (3.5)-(3.7) are specified only for $n>0.121 \sigma$.
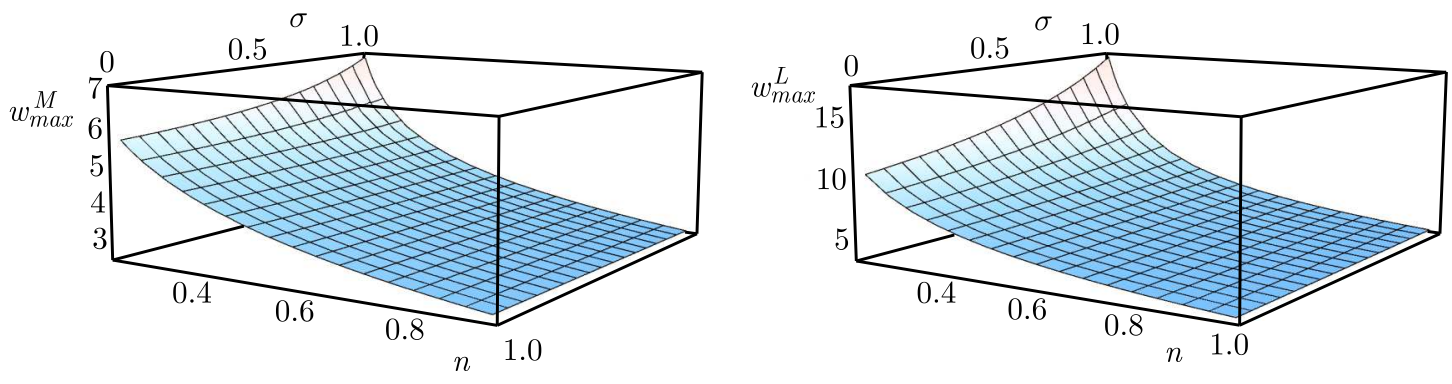

Fig. 6. Maximum deflection of the cantilever plate strip
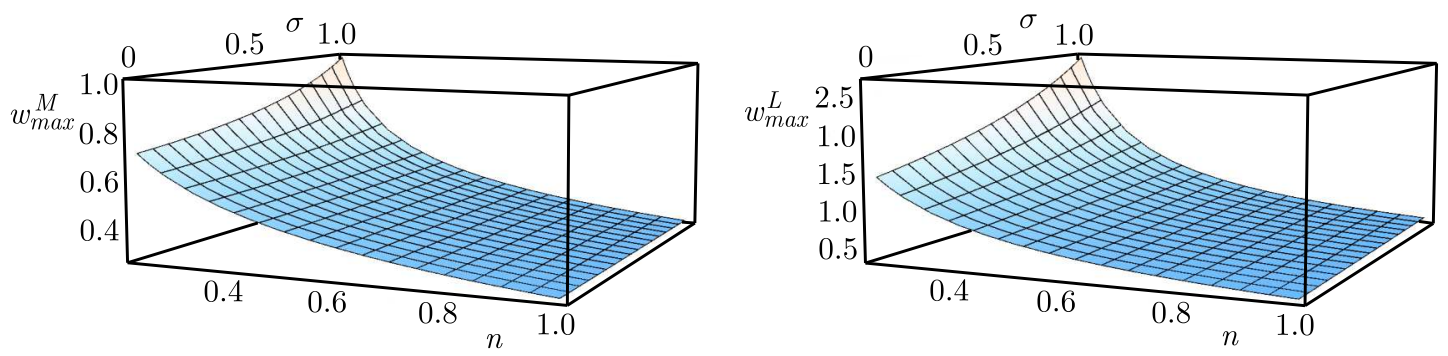

Fig. 7. Maximum deflection of the simple-supported plate strip

\section{Conclusions}

The paper proposes the application of the linear six-parameter shell theory to the analysis of orthotropic tensegrity plate-like structures. The continuum model of the plate is used. The 

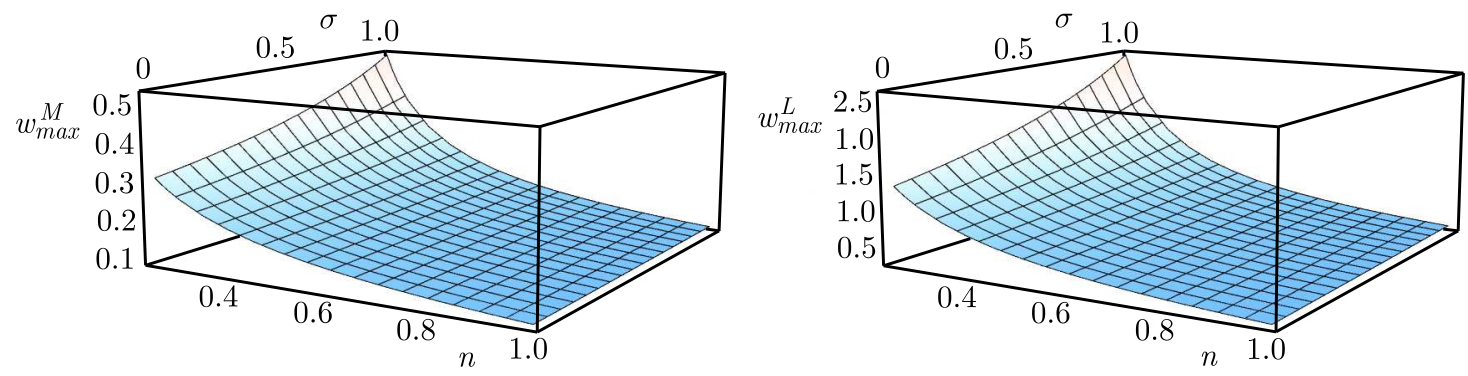

Fig. 8. Maximum deflection of the clamped-clamped plate strip

parametric analysis including the self-stress and selected geometrical parameters of tensegrity are considered. In the analysis, different planes of support of tensegrity plate-like structures are taken into account. The proposed approach helps one to understand unique properties and structural behaviour of tensegrities.

Comparing the obtained results, it can be noticed that displacements depend on the reference surface. Additionally, the influence of the self-stress level on the displacements also depends on the reference surface - is bigger when the lower (or upper) surface is considered. It means that the plane of support of tensegrity structures significantly affect the displacements. Furthermore, the results of parametric analysis shown that the influence of the self-stress level on the displacements decreases with an increase in the stiffness of cables (the parameter $n$ increases).

The closed form of the displacements and the internal forces obtained in the paper simplifies calculations. It is not necessary to describe the whole complex tensegrity structures with the use of computational methods. The closed formulas can be useful in the design process and construction of different types of tensegrity systems, such as beams, plates or more complex structures. Additionally, the obtained in the paper functions of the displacements and the internal forces can be used for analysis of orthotropic plate strips with any external loads and any boundary conditions.

\section{References}

1. Al Sabouni-Zawadzka A., Gilewski W., 2016, On orthotropic properties of tensegrity structures, Procedia Engineering, 153, 887-894

2. Al Sabouni-Zawadzka A., Gilewski W., KŁosowska J., Obara P., 2016, Continuum model of orthotropic tensegrity plate-like structures with self-stress included, Engineering Transactions, 64, 4, 501-508

3. Burzyński S., Chróścielewski J., Daszkiewicz K., Witkowski W., 2016, Geometrically nonlinear FEM analysis of FGM shells based on neutral physical surface approach in 6-parameter shell theory, Composities Part B, 107, 203-213

4. Chadwick P., Vianello M., Cowin S.C., 2001, A new proof that the number of linear elastic symmetries is eight, Journal of the Mechanics and Physics of Solids, 49, 2471-2492

5. Chróścielewski J., Kreja I., Sabik A., Witkowski W., 2011, Modeling of composite shells in 6-parameter nonlinear theory with driling degree of freedom, Mechanics of Advanced Materials and Structures, 18, 403-419

6. Chróścielewski J., Makowski J., Pietraszkiewicz W., 2004, Statics and Dynamics of Multifold Shell: Nonlinear Theory and Finite Element Method (in Polish), IPPT PAN, Warszawa

7. Chróścielewski J., Makowski J., Stumpf H., 1997, Finite element analysis of smooth, folded and multi-shell structures, Computer Methods in Applied Mechanics and Engineering, 41, 1-46 
8. Chróścielewski J., Pietraszkiewicz W., Witkowski W., 2000, On shear correction factors in the non-linear theory of elastic shells, International Journal of Solids and Structures, 47, $3537-3545$

9. Chróścielewski J., Pietraszkiewicz W., Witkowski W., 2016, Geometrical nonlinear FEM analysis of 6-parameter resultant shell theory based on 2-D Cosserat constitutive model, ZAMM, 96, 2, 191-204

10. Crawfordt, 2016, Transgender Architectonics: The Shape of Change in Modernist Space, Routledge, New York

11. Gilewski W., Kasprzak A., 2011, Tensegrities in bridge structures (in Polish), Acta Scientiarum Polonorum: Architectura, 10, 3, 35-43

12. Gilewski W., KŁosowska J., Obara P., 2015, Applications of tensegrity structures in civil engineering, Procedia Engineering, 111, 242-248

13. Gilewski W., KŁosowska J., Obara P., 2016, Verification of tensegrity properties of Kono structure and Blur Building, Procedia Engineering, 153, 173-179

14. Gómez-JÁuregui V., 2010, Tensegrity Structures and their Application to Architecture, Servicio de Publicaciones de la Universidad de Cantabria

15. Gómez-Jáuregui V., Arias R., Otero C., Manchado C., 2012, Novel technique for obtaining double-layer tensegrity grids, International Journal of Space Structures, 27, 2-3,155-166

16. Kebiche K., Kazi M., Aoual N., Motro R., 2008, Continuum model for systems in a self-stress state, International Journal of Space Structures, 23, 103-115

17. Khorshidi M.A., Shariati M., 2017, A multi-spring model for buckling analysis of cracked Timoshenko nanobeams based on modified couple stress theory, Journal of Theoretical and Applied Mechanics, 55, 4, 1127-1139

18. Kono Y., Choong K.K., Shimada T., Kunieda H., 1999, Experimental investigation of a type of double-layer tensegrity grids, Journal of the IASS, 40, 130, 103-111

19. Motro R., 2003, Tensegrity: Structural Systems for the Future, Kogan Page Science, London

20. Nowacki W., 1971, Theory of Asymmetrical Elasticity (in Polish), IPPT PAN, Warszawa

21. Obara P., Gilewski W., 2016, Dynamic stability of moderately thick beams and frames with the use of harmonic balance and perturbation methods, Bulletin of the Polish Academy of Sciences: Technical Sciences, 64, 4, 739-750

22. Pietraszkiewicz W., 1979, Finite Rotations and Lagrangean Description in The Non-linear Theory of Shells, Polish Scientific Publishers, Warszawa-Poznań

23. Pietraszkiewicz W., 2016, The resultant linear six-field theory of elastic shells: What it brings to the classical linear shell models?, ZAMM, 96, 8, 899-915

24. Schlaich M., 2004, The messeturm in Rostock - a tensegrity tower (in German), Journal of the IASS, 45, 145, 93-98

25. Skelton R.E., De Oliveira M.C., 2009, Tensegrity Systems, Springer, London

26. Timoshenko S.P., Gere J.M., 1961, Theory of Elastic Stability, McGraw-Hill, New York

27. Witkowski W., 2011, Synthesis of Formulation of Nonlinear Mechanics of Shells Undergoing Finite Rotations in the Context of FEM (in Polish), Wydawnictwo Politechniki Gdańskiej, Gdańsk

28. Woźniak C., 2001, Mechanics of Elastic Shells and Plates (in Polish), Polish Scientific Publishing House, Warszawa 